\title{
Size Dependent Heating Efficiency of Multicore Iron Oxide Particles in Low-Power Alternating Magnetic Fields
}

\author{
I.S. Smolkova*, N.E. Kazantseva, L. Vitkova, V. Babayan, J. Vilcakova, P. Smolka \\ Centre of Polymer Systems, University Institute, Tomas Bata University in Zlin, \\ Trida Tomase Bati 5678, 76001 Zlin, Czech Republic
}

\begin{abstract}
Aggregates of superparamagnetic nanoparticles, so called multicore particles get much attention due to collective magnetic behaviour. Despite the fact that saturation magnetization and coercivity of multicore particles are lower than for single particles of comparable size, they can generate large amount of heat in alternating magnetic field. This makes them promising for magnetic hyperthermia. However, correlation between internal magnetic structure of multicore particles and their heating ability in alternating magnetic fields are not clear yet. Detailed experimental investigations are required to determine the optimal sizes of multicore particles and the alternating magnetic field parameters to obtain maximal heat. In this study, we demonstrated how hydrodynamic size of multicore particles influences alternating magnetic field energy absorption. Dense aggregates composed of bare magnetic iron oxide nanoparticles of $13 \mathrm{~nm}$ were obtained by coprecipitation. Further peptization allowed to gain aqueous dispersions of multicore particles with various hydrodynamic size, varing from 85 to $170 \mathrm{~nm}$, due to electrostatic stabilization. Multicore particles dispersions have saturation magnetization of $40 \mathrm{~A} \mathrm{~m}^{2} / \mathrm{kg}_{\mathrm{Fe}_{3} \mathrm{O}_{4}}$ and coercivity of $79.6 \mathrm{~A} / \mathrm{m}$ regardless of their size. Dispersion of $85 \mathrm{~nm}$ multicore particles is stable and provides specific loss power of $42 \mathrm{~W} / \mathrm{g}_{\mathrm{Fe}}$. Further increase of hydrodynamic size leads to low stability and loss of the ability to generate heat in alternating magnetic field.
\end{abstract}

DOI: 10.12693/APhysPolA.131.663

PACS/topics: 75.47.Lx, 75.50.Mm, 75.60.Jk, 75.75.-c, 75.78.-n

\section{Introduction}

Superparamagnetic (SPM) iron oxide nanoparticles are nowadays finding application for selective cancer treatment by magnetic hyperthermia. In this method the tumor is heated by the embedded magnetic material to $42-45^{\circ} \mathrm{C}$ that induce selective cytotoxicity. The heating ability of the magnetic material exposed to alternating magnetic field (AMF) is characterized by specific loss power (SLP). Generally, two types of mechanisms account for heat generation: (i) the Néel relaxation of magnetic moment within the particle and (ii) the Brown relaxation of the whole particle. SPM nanoparticles with low energy barrier of magnetization reversal easily rotate toward field direction without any heat losses. However, when SPM nanoparticles are gathered into aggregates due to interparticle magnetic interaction, the energy barrier of magnetization reversal increases leading to production of substantial heat when exposed to AMF [1]. These aggregates of SPM nanoparticles are called multicore particles (MCPs). The individual magnetic moments of iron oxide cores make up an effective magnetic moment of the MCP and it displays ferromagnetic behavior with non-zero hysteresis [2]. Unfortunately, there is no theory describing the magnetization dynamics of such materials so far [3]. However, it is obvious that the main parameters influencing the heating efficiency in AMFs are shape, size and size distribution of iron oxide cores and

*corresponding author; e-mail: smolkova@cps.utb.cz multicores themselves as well as the concentration in the dispersion medium. In this study, we obtained MCPs with various hydrodynamic size composed of magnetic iron oxide cores of $13 \mathrm{~nm}$ and compared their magnetostructural properties with the heating efficiency in AMF.

\section{Experimental}

\subsection{Synthesis of iron oxide magnetic nanoparticles}

Iron oxide magnetic nanoparticles were prepared by co-precipitation method according to $[4,5]$. Briefly, solution of iron (II) and iron (III) chlorides with molar ratio $\mathrm{Fe}^{2+}: \mathrm{Fe}^{3+}=1: 2$ was slowly added into the ammonia solution $(0.38 \mathrm{M})$ with continuous heating to $70^{\circ} \mathrm{C}$ and stirring at $700 \mathrm{rpm}$. After the whole solution was added, the mixture was kept at $70^{\circ} \mathrm{C}$ and stirred for one hour. The whole reaction was conducted under argon atmosphere. The prepared dark-brown sediment of magnetic nanoparticles was then decanted and washed with demineralized water.

\subsection{Peptization of nanoparticles and obtaining of $M C P s$}

In order to obtain dispersion of MCPs in water, the iron oxide nanoparticles sediment was mixed with $0.001 \mathrm{M} \mathrm{HCl}$ until the $\mathrm{pH}$ of the dispersion reached 2.7 and ultrasonicated. After this, dark-brown supernatant was observed and collected by holding the remaining sediment with magnet. Further addition of water to the remaining sediment also resulted in the formation of supernatant with dispersed MCPs. Peptization was observed during dilution until $\mathrm{pH} 5$. To obtain the dispersions of MCPs in agar matrix, we added $3.4 \mathrm{wt} \%$ of agarose to 
the water dispersion of MCPs and heated under continuous stirring to $70^{\circ} \mathrm{C}$. After this the mixture was placed in a fridge where the solid matrix was quickly formed.

\subsection{Characterization}

Average size and polydispersity of nanoparticles was evaluated from transmission electron microscopy (TEM) on JEOL JEM - 2100F. Hydrodymanic size of MCPs and zeta potential were measured by dynamic light scattering (DLS) and laser doppler velocimetry on Zetasizer Nano ZS, Malvern Instruments. The concentration of iron oxide in dispersions and iron content was determined by energy dispersive X-ray fluorescence spectroscopy by ARL Quant'X EDXRF Analyzer, Thermo Scientific. The magnetostatic properties were measured with vibrating sample magnetometer Lake Shore 7407 at room temperature in air atmosphere in a magnetic field of up to $796 \mathrm{kA} / \mathrm{m}$. Home-made AMF generator was used to determine the SLP of the samples. It consisted of a signal generator Agilent 33521A, RF broadband amplifier AR $\mathrm{RF} /$ Microwave Instrumentation $800 \mathrm{~A} 3 \mathrm{~A}$, induction coil (90 $\mathrm{mm}$ diameter), interchangeable capacitors and magnetic field sensor. The measurements were carried out at AMF of $1048 \mathrm{kHz}$ frequency and $5.9 \mathrm{kA} / \mathrm{m}$ amplitude. The temperature was measured with monitoring system ReFlex 4, Neoptix and fibre optic temperature sensor T1S-03-PT06 inserted directly in dispersion. SLP was calculated according to equation: $\mathrm{SLP}=(\mathrm{d} T / \mathrm{d} t)(c / m)$, where $T$ is temperature, $t$ is time, $c$ is heat capacity and $m$ is mass of iron in the dispersion.

\section{Results and discussion}

For the synthesis of iron oxide magnetic nanoparticles we applied coprecipitation method with synthesis parameters verified in our previous studies, that guarantee formation of uniform, highly crystalline nanoparticles $[4,5]$. Obtained nanoparticles have average size of $13 \mathrm{~nm}$ and polydispersity 0.3 according to TEM investigations (Fig. 1) and therefore are smaller than the critical SPM diameter for magnetite and maghemite [1]. Magnetic nanoparticles represent a mixture of magnetite, non-stoichiometric magnetite and maghemite nanocrystals according to X-ray diffraction and Mössbauer spectroscopy [4].

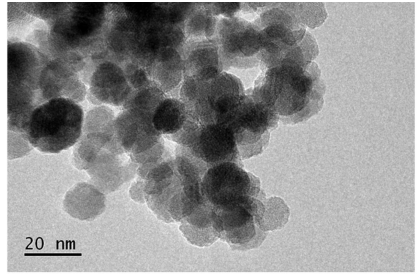

(a)

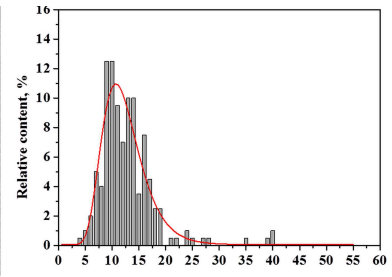

(b)
Fig. 1. TEM of magnetic iron oxide nanoparticles (cores): (a) original sample, (b) particle size distribution. Adapted from [4] with permission of Elsevier.
As no other substances than iron salts and ammonia solutions were used during synthesis, the obtained nanoparticles are bare and gather in aggregates due to magnetic interparticle interaction. These aggregates have a broad size distribution, i.e. their size can be of tens nanometers to several $\mu \mathrm{m}$, and therefore they do not form stable dispersions in water. After the synthesis, when the medium $\mathrm{pH}$ is 8.5 , aggregated nanoparticles sediment on the bottom of the flask (Fig. 2a).

When precipitate was treated with the diluted solution of hydrochloric acid $(0.001 \mathrm{M})$ to $\mathrm{pH}$ of 2.7 , the formation of dark brown supernatant was observed, indicating the peptization of nanoparticles aggregates (Fig. 1b). Treatment by hydrochloric acid gives the amphoteric magnetite surface the positive charge according to protolytic reaction [6]: $\mathrm{Fe}-\mathrm{OH}+\mathrm{H}^{+} \leftrightarrow \mathrm{Fe}-\mathrm{OH}_{2}^{+}$.

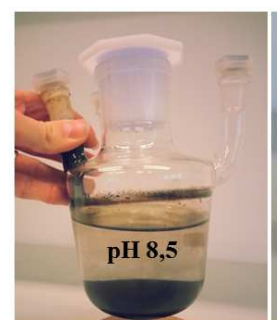

(a)

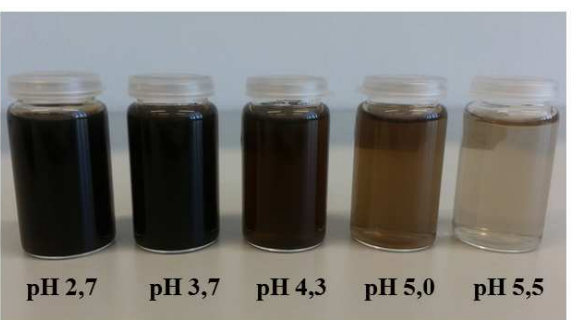

(b)
Fig. 2. Images of: (a) magnetic nanoparticles after synthesis, sedimented at the bottom of the flask, (b) supernatants obtained by peptization, representing a water dispersions of MCPs with various hydrodynamic size at different $\mathrm{pH}$.

DLS investigations of supernatant revealed that it contains MCPs of $85 \mathrm{~nm}$ (Fig. 3). MCPs demonstrate high positive surface charge that provides their mutual repulsion and stability of the system. Indeed, particles in the supernatant do not sediment even when exposed to a strong permanent magnet.

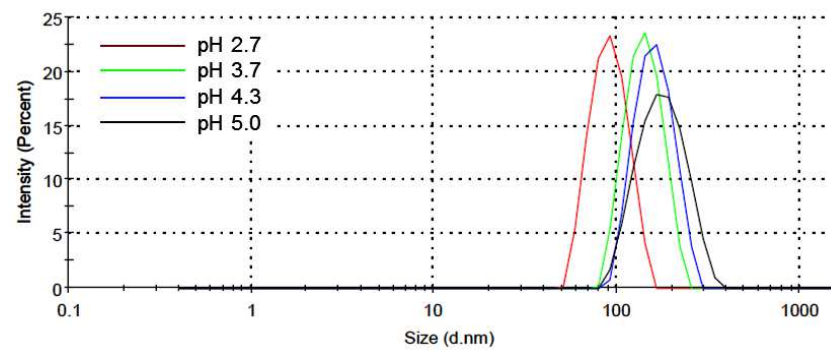

Fig. 3. Intensity-weighted DLS data of MCPs aqueous dispersions.

After removal of supernatant and dilution of the remaining sediment with water a new supernatant layer arises. It has $\mathrm{pH}$ of 3.7, dispersed MCPs are of $140 \mathrm{~nm}$ and have zeta potential of $+40 \mathrm{mV}$. With further dilution and $\mathrm{pH}$ decrease, the size of MCPs increases and the surface 
charge decreases (Fig. 3, Table I). Formation of MCPs with various hydrodynamic size at different $\mathrm{pH}$ is associated with the competition between attractive magnetic interactions (dipolar and exchange interactions) and repulsive electrostatic interactions. With an increase of $\mathrm{pH}$ of dispersion media, the electrostatic repulsion lowers, magnetic cores aggregate and as a result, the average size of MCPs increases. The polydispersity, however is the same regardless MCPs size and is of 0.1 . When $\mathrm{pH}$ approaches the isoelectric point of magnetite (at $\mathrm{pH}>5.5$ ), the surface charge is low, and the size of the particles is large, which does not allow them to be in the dispersed state. In this case, the peptization does not occur and the supernatant is transparent.

Characteristics of supernatants at different $\mathrm{pH}$ values.

TABLE I

\begin{tabular}{|c|c|c|c|c|c|c|}
\hline \multirow[t]{2}{*}{$\mathrm{pH}$} & \multirow{2}{*}{$\begin{array}{c}\text { Iron content } \\
\text { [wt } \%]\end{array}$} & \multirow{2}{*}{$\begin{array}{l}\text { Size } \\
{[\mathrm{nm}]}\end{array}$} & \multirow{2}{*}{$\begin{array}{c}\text { Zeta potential } \\
{[\mathrm{mV}]}\end{array}$} & \multirow[t]{2}{*}{ Stability } & SLP in water & SLP in agar \\
\hline & & & & & \multicolumn{2}{|c|}{$\left[\mathrm{W} / \mathrm{g}_{\mathrm{Fe}}\right]$} \\
\hline 2.7 & 0.037 & 85 & +44 & stable & $42 \pm 5$ & $28 \pm 6$ \\
\hline 3.7 & 0.036 & 140 & +42 & stable for 3 days & $25 \pm 4$ & $23 \pm 4$ \\
\hline 4.3 & 0.025 & 160 & +41 & stable for 2 days & $8 \pm 3$ & $9 \pm 3$ \\
\hline 5.0 & 0.012 & 170 & +29 & stable for 1 day & 0 & 0 \\
\hline
\end{tabular}

Study of magnetic properties of the dispersions revealed that MCPs have saturation magnetization of $40 \mathrm{~A} \mathrm{~m}^{2} / \mathrm{kg}_{\mathrm{Fe}_{3} \mathrm{O}_{4}}$ and coercivity of about $79.6 \mathrm{~A} / \mathrm{m}$ regardless of their size. The dispersions, however, display quite different heat outcome under exposure to AMF of $1048 \mathrm{kHz}$ and $5.8 \mathrm{kA} / \mathrm{m}$ (Table I). SLP increases with the decrease of MCPs size. The water dispersion of $85 \mathrm{~nm}$ MCPs displays the highest SLP of $42 \mathrm{~W} / \mathrm{g}_{\mathrm{Fe}}$. In the literature considering systems of MCPs, the difference in SLP is explained by acceleration of the Brown relaxation with the increase of hydrodynamic volume of MCPs [7]. To verify this statement we performed the SLP measurements on the dispersions of MCPs in viscous agar matrix, that inhibits the Brown relaxation. As can be seen from Table I, only for MCPs of $85 \mathrm{~nm}$ there is a clear difference between heat outcome in media with different viscosity. Therefore, the Brown relaxation indeed contributes to the magnetic losses and thus increases the heating efficiency of the dispersion of $85 \mathrm{~nm}$ MCPs in water. However, it should be noted that, right after the synthesis, aggregates of iron oxide nanoparticles with broad size distribution demonstrate SLP of $8 \pm 1 \mathrm{~W} / \mathrm{g}_{\mathrm{Fe}}$ in agar matrix. Therefore, fractionation of MCPs is required to increase the SLP.

\section{Conclusions}

Aqueous dispersions of MCPs with various hydrodynamic size were obtained by peptization of coprecipitated bare iron oxide nanoparticles of $13 \mathrm{~nm}$. Protonation of magnetite surface leads to positive charge and as a result, electrostatic repulsive interactions dominate over attractive magnetic interactions ensuring dispersion stability. With increase of $\mathrm{pH}$ up to 5 , the average size of MCPs increases and the dispersion stability decreases. Though obtained dispersions display saturation magnetization of $40 \mathrm{~A} \mathrm{~m} \mathrm{~m}^{2} / \mathrm{kg}_{\mathrm{Fe}_{3} \mathrm{O}_{4}}$ and coercivity of about $79.6 \mathrm{~A} / \mathrm{m}$ regardless of MCPs size, their heating ability in AMF is quite different. The highest heat outcome displays the dispersion of MCPs with hydrodynamic size of $85 \mathrm{~nm}$ due to the effect of both the Néel and Brown relaxation. An increase in the dispersion viscosity leads to the inhibition of the Brown relaxation; this was demonstrated for the dispersion of $85 \mathrm{~nm}$ MCPs in an agar matrix; in this case, SLP decreased from $42 \mathrm{~W} / \mathrm{g}_{\mathrm{Fe}}$ to $28 \mathrm{~W} / \mathrm{g}_{\mathrm{Fe}}$. Nevertheless, the SLP value for both aqueous and agar dispersions of $85 \mathrm{~nm}$ MCPs are much higher compared to non-fractionated MCPs, indicating the importance of fractionation step in the preparation of iron oxides for magnetic hyperthermia.

\section{Acknowledgments}

This work was supported by the Ministry of Education, Youth and Sports of the Czech Republic - Program NPU I (LO1504).

\section{References}

[1] S. Dutz, R. Hergt, Nanotechnology 25, 452001 (2014).

[2] C.L. Dennis, K.L. Krycka, J.A. Borchers, R.D. Desautels, J. van Lierop, N.F. Huls, A.J. Jackson, C. Gruettner, R. Ivkov, Adv. Funct. Mater. 25, 4300 (2015).

[3] A.Y. Zubarev, A.F. Abu-Bakr, L.Y. Iskakova, S.V. Bulycheva, Magnetohydrodynamics 51, 647 (2015).

[4] I.S. Smolkova, N.E. Kazantseva, V. Babayan, P. Smolka, H. Parmar, J. Vilcakova, O. Schneeweiss, N. Pizurova, J. Magn. Magn. Mater. 374, 508 (2015).

[5] I.S. Smolkova, N.E. Kazantseva, H. Parmar, V. Babayan, P. Smolka, P. Saha, Mater. Chem. Phys. 155, $178(2015)$.

[6] E. Illes, E. Tombacz, J. Coll. Interf. Sci. 295, 115 (2006).

[7] F. Arteaga-Cardona, K. Rojas-Rojas, R. Costo, M.A. Mendez-Rojas, A. Hernando, P. de la Presa, J. Alloys Comp. 663, 636 (2016). 